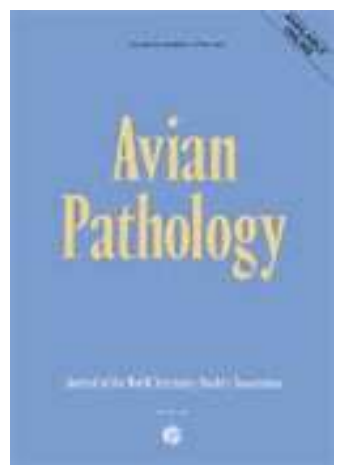

\title{
Quantification of gut lesions in a subclinical necrotic enteritis model
}

\begin{tabular}{|r|l|}
\hline Journal: & Avian Pathology \\
\hline Manuscript ID: & CAVP-2007-0026.R1 \\
\hline Manuscript Type: & Original Research Paper \\
\hline Date Submitted by the \\
Author: & 26-Apr-2007 \\
\hline Kumplete List of Authors: & $\begin{array}{l}\text { Gholamiandekhordi, Ahmad R. } \\
\text { Timbermont, Leen } \\
\text { Lanckriet, Anouk } \\
\text { vandenbroeck, wim } \\
\text { Pedersen, Karl } \\
\text { Dewulf, Jeroen; Ghent University } \\
\text { Pasmans, Frank; Ghent University, Pathology, Bacteriology and } \\
\text { Avian Diseases } \\
\text { Haesebrouck, Freddy } \\
\text { Ducatelle, Richard } \\
\text { Van Immerseel, Filip; Ghent University, Pathology, Bacteriology and } \\
\text { Avian Diseases }\end{array}$ \\
\hline Keywords & \begin{tabular}{l} 
necrotic enteritis, clostridium perfringens, gut damage , histology \\
\hline
\end{tabular} \\
\hline
\end{tabular}

\section{SCHOLARONE \\ Manuscripts}


Quantification of gut lesions in a subclinical necrotic enteritis model

\author{
Ahmad R. Gholamiandekhordi ${ }^{1,5}$, Leen Timbermont ${ }^{1}$, Anouk Lanckriet ${ }^{1}$, Wim Van Den \\ Broeck $^{2}$, KarlPedersen ${ }^{3}$, Jeroen Dewulf ${ }^{4}$, Frank Pasmans ${ }^{1}$, Freddy Haesebrouck ${ }^{1}$, Richard \\ Ducatelle $^{1}$, Filip Van Immerseel ${ }^{1, *}$
}

${ }^{1}$ Research Group Veterinary Public Health and Zoonoses, Department of Pathology, Bacteriology and Avian Diseases, Faculty of Veterinary Medicine, Ghent University, Salisburylaan 133, B-9820 Merelbeke, Belgium. ${ }^{2}$ Department of Morphology, Faculty of Veterinary Medicine, Ghent University, Salisburylaan 133, B-9820 Merelbeke, Belgium. ${ }^{3}$ Department of Poultry, Fish and Fur animals, Danish Institute for Food and Veterinary Research, Hangoevej 2, DK-8200 Aarhus,

Denmark. ${ }^{4}$ Research Group Veterinary Public Health and Zoonoses, Department of Reproduction, Obstetrics and Herd Health, Faculty of Veterinary Medicine, Ghent University, Salisburylaan 133, B-9820 Merelbeke, Belgium. ${ }^{5}$ Department of Clinical Sciences, Faculty of Veterinary Medicine, Shahrekord University, Saman Road, PO Box 115, Shahrekord, Iran

Short title: Gut lesions in necrotic enteritis model

Received: 21 February 2007

*Corresponding author: Tel. +3292 647748; fax: +3292647494.

E-mail address: filp.vanimmerseel@Ugent.be 


\title{
Cavp-2007-0026.R1
}

Quantification of gut lesions in a subclinical necrotic enteritis model

\author{
Ahmad R. Gholamiandekhordi ${ }^{1,5}$, Leen Timbermont ${ }^{1}$, Anouk Lanckriet ${ }^{1}$, Wim Van Den \\ Broeck $^{2}$, KarlPedersen ${ }^{3}$, Jeroen Dewulf ${ }^{4}$, Frank Pasmans ${ }^{1}$, Freddy Haesebrouck ${ }^{1}$, Richard \\ Ducatelle $^{1}$, Filip Van Immerseel ${ }^{1, *}$
}

\begin{abstract}
Currently Clostridium perfringens induced necrotic enteritis is a major problem in broiler flocks. In the present study, broilers were inoculated with a combination of Eimeria maxima or overdose coccidial vaccine (one inoculation) with $C$. perfringens (repeated inoculations). Single $C$. perfringens, E. maxima or an overdose of live coccidial vaccine inoculations did not result in grossly visible necrotic gut lesions, while combined inoculation resulted in typical necrotic lesions at approximately 4 days after inoculations with $C$. perfringens in approximately half of the inoculated animals. Semi-quantitative histological lesion scoring was done to evaluate gut damage in gut sections of animals in which no gross necrotic lesions were detected. This included scoring of hyperaemia, haemorrhages, the amount of red blood cells and protein precipitate in the lumen, villus fusion and epithelial defects. Villus length and villus length/crypt depth ratio were also analysed. This approach proved to be discriminative between single E. maxima infection, overdose of coccidial vaccine or $C$. perfringens inoculations and the non-inoculated control group, and between the double and single inoculated groups. In general, highest histological scores for gut lesions were observed in the double inoculated groups, but the single inoculated groups had higher scores than the control group. It was concluded that oral inoculation of broilers with an overdose of live coccidial vaccine in combination with multiple oral $C$. perfringens inoculations is a suitable model for necrotic enteritis without inducing mortality of the animals. C. perfringens and Eimeria act synergistically in inducing grossly visible gut damage.
\end{abstract}




\section{Introduction}

Clostridium perfringens causes a variety of economically significant diseases in domestic animals (Songer, 1996). A major problem currently is necrotic enteritis in poultry (Van Immerseel et al. 2004; Williams, 2005). Antimicrobials, commonly used to increase weight gain in broiler chickens, were banned from poultry feed in Europe from January 1, 2006. The ban on use of these growth promoting antibiotics from broiler feed is a factor that inevitably changes the bacterial microflora in the intestinal tract of broiler chickens (Knarreborg et al., 2002) and it has become clear that ceasing to use these antibiotic growth promoters has increased the prevalence of health problems in broiler flocks, particularly problems of retarded growth and of C. perfringens associated necrotic enteritis (Williams, 2005).

C. perfringens infections in poultry may present as acute clinical disease or may be subclinical. The acute form of the disease is named necrotic enteritis. It leads to increased mortality in the broiler flocks, caused by extensive necrosis of the small intestinal mucosa (Wages and Opengart, 2003). In the subclinical form, damage to the intestinal mucosa caused by $C$. perfringens leads to decreased digestion and absorption, reduced weight gain and increased feed conversion ratio (Elwinger et al., 1992; Kaldhusdal et al., 2001).

Many attempts to reproduce necrotic enteritis in poultry have been described in the literature, using many different infection protocols, most of which use predisposing factors to induce disease. The most well-known predisposing factors are coccidial infection and high protein diets. Inoculations with Eimeria spp. combined with $C$. perfringens are used in the majority of protocols (Collier et al., 2003; Williams et al., 2003).

There is a much variation in severity of the necrotic enteritis induced in the different models, some inducing only very low levels of gut necrosis and thus very low lesions scores in only a minority of treated animals. Such models can be used to study subclinical disease (Kaldhusdal \& Hofshagen, 1992). Parameters that are used to measure subclinical disease are body weight gain and 
feed conversion ratio, meaning that large groups are needed to show statistically significant differences. Other models induce severe gut necrosis and a high rate of mortality and are suitable to study clinical necrotic enteritis (Riddell \& Kong, 1992) but raise ethical questions.

The ideal model to study the pathogenesis of necrotic enteritis and the related subclinical disease is one in which a high percentage of the treated animals develop grossly visible necrosis of the intestinal mucosa, but without mortality. Moreover, in an ideal model, the gut should not be overwhelmed by coccidial pathogens interfering with histological analysis of the gut samples. There is also a need for reliable parameters that can be used to measure $C$. perfringens-induced subclinical disease. In the study described below, a model is presented with these characteristics using an overdose of an anticoccidial vaccine, combined with oral $C$. perfringens inoculations. This model is compared with models using Eimeria and the differences are discussed. Furthermore, the histological parameters are scored, allowing a semi-quantitative evaluation of gut damage, aiming to provide a system that detects mild $C$. perfringens-associated gut damage in birds without specific gross necrotic enteritis lesions.

\section{Materials and methods}

Vaccines and strains. C. perfringens strain 56 was isolated from the gut of a broiler chicken with severe necrotic gut lesions from a flock with weight gain problems. The strain was characterized using previously described methods (Gholamiandehkordi et al., 2006) and was classified as a type A strain (no beta2 or enterotoxin genes) and produces moderate amounts of alpha toxin in vitro. The strain was made rifampicin resistant by plating on Columbia blood agar (Oxoid, Basingstoke, England) containing increasing concentrations of the antibiotic. This was done to provide a strain for use further studies that are planned. Before inoculation of the birds, the bacteria were cultured 
for $24 \mathrm{~h}$ in Brain Heart Infusion (BHI, Oxoid, Basingstoke, England) broth containing $20 \mu \mathrm{g}$ per ml rifampicin.

The anticoccidial vaccine Paracox ${ }^{\mathrm{TM}}$ (Schering-Plough Animal Health, Brussels, Belgium), containing live, attenuated oocysts of Eimeria acervulina (two lines), Eimeria maxima (two lines), Eimeria mitis, Eimeria necatrix, Eimeria praecox and Eimeria tenella was used in this study. The Eimeria strain used was E. maxima (Weybridge strain), kindly provided by Dr. R. Marshall (Veterinary Laboratories Agency, Weybridge, UK). The commercial Gumboro vaccine, Nobilis Gumboro D78 (Intervet, Mechelen, Belgium), was used.

Birds and housing . All trials used non-vaccinated Ross 308 broiler chickens of mixed sex that were obtained at one-day-old from a commercial hatchery. All treatment groups were housed in the same room, in individual cages of $1 \mathrm{~m}^{2}$, on a litter floor. Each cage was separated by a solid wall to prevent contact between birds from different treatment groups. In each trial (see below) one single group of birds was housed together in one cage for each treatment. Density of the birds was 14 per $\mathrm{m}^{2}$ for all groups in Trial 1, 25 per $\mathrm{m}^{2}$ for all groups in Trial 2 and for groups 2 and 4 of Trial 3, and 28 for groups 1 and 3 of Trial 3. Before each trial, the isolation rooms were decontaminated by formaldehyde fumigation and application of a commercial anticoccidial disinfectant. Fifty-six, 150 and 100 chickens were reared in Trials 1, 2 and 3, respectively. Due to a low level of mortality, not all were included in the results, and the above mentioned density could slightly differ. They were given drinking water and feed ad libitum. A 23/1 light/darkness programme was applied. All experiments were performed under supervision of the ethical committee of the Faculty of Veterinary Medicine, Ghent University.

Experimental study design. Three experimental in vivo trials were carried out. The important characteristics of the trials are shown in Table 1. The feed was a meal feed and its composition is shown in Table 2. 
Trial 1. Trial 1 was carried out to determine the optimal time point at which lesions could be detected. Birds were divided in four groups of 14 chickens. The diet was wheat/barley $(50 \% / 10 \%)$ based, with soybean meal as the protein source. From day 19 onwards, the same (isocaloric) diet was used with the exception that fishmeal (30\%) was used as the protein source. All birds of groups 1 and 2 were challenged orally three times a day (i.e. at 09:00, 13:00 and 17:00) with approximately $4 \times 10^{8}$ colony-forming units (cfu) of $C$. perfringens strain 56 on days 18, 19, 20 and 21, using a plastic tube. On day 19, birds of group 1 and 3 were individually inoculated orally with a 10-fold dose of Paracox, using a plastic tube. On day 18 two animals per group were euthanized by intravenous injection of T61 (Intervet, Mechelen, Belgium). For days 21, 25 and 27 the number of animals per treatment group that was euthanized is shown in Table 4. Necrotic enteritis lesion scoring and sample collection was carried out as described below.

Trial 2. The experimental protocol was slightly different from Trial 1 (Table 1). A virulent $E$. maxima strain was used and compared to the Paracox inoculation as described in Trial 1, as described in other models using the same E. maxima strain (Williams et al., 2003). Birds were divided in six groups of 25 animals. The diet of the animals was a wheat/rye $(43 \% / 7.5 \%)$ based diet, with soybean meal as the protein source. From day 20 onwards, the same diet was used except that fishmeal (30\%) was used as the protein source. Gumboro vaccine was given in the drinking water at day 18 to all six groups, since this could cause immunosuppression and thus be a predisposing factor for development of necrotic enteritis (McReynolds et al., 2004). . All birds of groups 1, 2 and 3 were challenged orally three times a day with approximately $4 \times 10^{8} \mathrm{cfu}$ of $C$. perfringens strain 56 on days 19,20, 21 and 22. On day 20, birds of group 2 and 5 were inoculated orally with a 10fold dose of Paracox, while birds of group 1 and 4 were challenged orally with 30,000 oocysts of $E$. maxima. On day 19 two birds from each group were euthanized by intravenous injection of T61. Table 4 shows the numbers of animals per treatment group that were euthanized on days 21, 22, 23, 
24, 25 and 26. Necrotic enteritis lesion scoring and sample collection was performed, as described below.

Trial 3. The diet of the birds was wheat/rye (43\%/7.5\%) based, with soybean meal as the protein source. Birds were divided in four groups of 26 animals. From day 20 onwards, the same diet was used except that fishmeal (30\%) was the protein source in groups 1 and 2, and soybean meal and fishmeal (5\%) in groups 3 and 4 . All groups were challenged three times a day with approximately $4 \times 10^{8}$ cfu $C$. perfringens strain 56 on days 19, 20, 21 and 22. On day 16, birds of groups 2 and 4 were inoculated orally with a 10-fold dose of Paracox, while this was done at day 20 for groups 1 and 3. Gumboro vaccine was given to all groups in the drinking water at day 18 . On day 16 two animals from groups 2 and 4, and at day 19 two animals of each group were euthanized. From 21 to 27 days, the numbers of animals euthanized as can be seen in Table 4. No negative control groups were included in this trial since the aim was to compare different $C$. perfringens inoculation regimes and different protein levels in the feed. Necrotic enteritis lesion scoring and sample collection was performed as described below.

Macroscopic lesion scoring. Inspection of the mucosa was done after gently removing the gut contents with cotton swabs, when necessary. Post mortem examination for necrotic enteritis lesions was performed using a modification of the grading method of Truscott \& Al-Sheikhly (1977). Lesions were graded from $0-3$, with $0=$ no lesions; $1=$ focal necrosis and ulceration; $2=$ patches of necrosis 2-3 cm long; and $3=$ diffuse necrosis typical of field cases. The score used by Truscott $\&$ Al-Sheikhly for 'thin walled and friable intestines' was not applied her because of the difficulties in scoring this characteristic objectively, and due to the lack of scientific documentation of an association between 'thin walled and friable intestines' and necrotic enteritis. 
Histopathology and morphometry. Samples of duodenum, jejunum (proximal to Meckel's diverticulum) and ileum (proximal to ileo-caecal junction) of all the birds examined were fixed in phosphate buffered formalin for at least $24 \mathrm{~h}$, after which they were embedded in paraffin. Sections of $5 \mu \mathrm{m}$ were cut and stained with haematoxylin and eosin.

All samples from Trials 1 and 2, including those with necrotic enteritis lesions, were used for villus length and crypt depth measurement. The numbers sampled can be seen in Table 4 . Measurements were not taken in Trial 3. Villus length and crypt depth were dtermined by random measurement of 10 villi/crypts per section (one section per gut region per bird) using a PC-based image analysis system, Olympus BX61 Digital Camera DP50 (Olympus NV, Aartselaar, Belgium) with software Analysis ${ }^{\circledR} \mathrm{J}-2$. The ratio villus length/crypt depth was determined and the mean was calculated for each gut segment, and for each treatment group.

To further quantify histological observations, scoring systems were developed for different parameters, i.e. for villus fusion, congestion or dilation of blood vessels, capillary haemorrhages, red blood cells in the gut lumen, proteinaceous material in the gut lumen and epithelial cell defects. The same samples were analyzed as for villus length and crypt depth measurements, so the numbers of birds sampled can be seen in Table 4. Again, measurements were not made in Trial 3. A description of the scoring system is shown in Table 3.

Statistical analysis. In each trial, comparison of the numbers of birds with macroscopic necrotic enteritis lesions in the different groups was done by means of logistic regression. For analysis of differences between the groups in the mean macroscopic necrotic enteritis lesions scores in Trial 3, the scores were first dichotomized (present: score $>0$; absent: score $=0$ ) since they were totally non-normally distributed and subsequently analyzed by means of logistic regression. The difference between groups in villus length/crypt depth ratio (V/C ratio) was analysed by means of analysis of variance. The difference between groups in total score of gut damage for Trial 2 was analysed by 
means of multivariable analysis of variance. In both the multivariable and univariable analysis of variance Sheffé post-hoc tests were used. All analyses were done by means of SPSS 14.0.

\section{Results}

Macroscopic observations. Table 4 shows the number of birds with grossly visible necrotic lesions for all trials. These lesions presented as multiple foci, mostly in the jejunum (Figure 1). No birds sampled before day 21 had gross lesions, in any of the trials.

In Trial 1, only the animals that were inoculated with both $C$. perfringens and a 10 -fold dose of Paracox, developed lesions (group 1). The lesions were detected 4 days after the final $C$. perfringens inoculation. After a further two days lesions were no longer detected. Sampling was not done for the first 3 days after C. perfringens inoculations. No gross lesions were observed in the control group (group 4) or in the groups inoculated with a 10-fold dose of Paracox (group 3), or with $C$. perfringens only (group 2). Overall, no significant differences between the groups were found in the proportion of animals developing gross lesions.

In Trial 2 lesions were detected only in the groups that were inoculated with $C$. perfringens, combined with E. maxima (group 1) or with a 10-fold dose of Paracox (group 2). Lesions were first detected 2 days after the final C. perfringens inoculations. Again no significant differences between the groups were found in the proportion of animals developing gross lesions. In Trial 3 the number of sampled birds developing necrotic lesions ranged between 30 and $62 \%$ in the different groups, and the time interval during which lesions were detected was longer (Table 4). Lesions were already detected on the last day of $C$. perfringens inoculations. Overall, a significant $(p=0.045)$ difference in the proportion of animals developing gross lesions was found between the groups in Trial 3.

Table 5 shows the mean necrotic enteritis lesion scores for Trial 3. Generally, lesion scores were highest in the jejunum and rather low in the ileum. The group inoculated with a 10-fold dose 
of Paracox on day 20, and inoculated with C. perfringens on day 19, 20, 21 and 22 (group 1), generally had the highest lesion scores. For the duodenum, no significant differences were found between the groups in the proportion of animals showing necrotic enteritis lesions. For the jejunum a borderline non-significant $(\mathrm{p}=0.066)$ difference was found, whereas for the ileum a significant ( $\mathrm{p}$ $=0.043$ ) difference was found between the groups.

Microscopic observations. Necrotic enteritis lesions were typically visible microscopically as severe necrosis of the intestinal mucosa, with an abundance of fibrin admixed with cellular debris adherent to the necrotic mucosa, in which large clusters of bacteria were present (Figure 2). These lesions were observed only in groups receiving repeated $C$. perfringens inoculations combined with either Paracox or E. maxima inoculations. Marked infiltration of heterophilic granulocytes was also observed. In areas without macroscopically visible necrosis in groups inoculated with $C$.

perfringens (either in combination with Paracox or E. maxima or not) large numbers of rod-shaped bacteria were seen adhering to aggregates of protein in the lumen between the villi (Figure 3).

Tables 6 and 7 show the ratio villus length/crypt depth (V/C ratio) for duodenal and jejunal sections in Trial 1 and for duodenal, jejunal and ileal sections in Trial 2. Statistical differences between the groups for all gut sections and time points are indicated in Tables 6 and 7.

In Trial 1, duodenal sections of non-inoculated animals (group 4) had the highest V/C ratio, while the duodenal sections of the animals inoculated with both $C$. perfringens and a 10-fold dose of Paracox (group 1) had the lowest. Also the animals receiving only $C$. perfringens or a 10-fold dose of Paracox (group 2 and 3) had lower V/C ratios than the control animals (group 4). Although the differences were less clear in the jejunal sections, V/C ratios of control animals (group 4) were higher than in the other treatment groups, and the group inoculated with both $C$. perfringens and a 10-fold dose of Paracox (group 3) tended to have the lowest V/C ratios.

In Trial 2, again the control group (group 6) had highest V/C ratios in all gut sections, compared with all other groups. E. maxima inoculations, either combined or not with $C$. 
perfringens, had a serious impact on the V/C ratio, especially in the ileum. All treatments, including the group that received a 10-fold dose of Paracox (group 5), and the group that was inoculated with $C$. perfringens (group 3) had decreased V/C ratios compared to the controls.

For duodenal and jejunal sections, generally highest gut damage parameter scores (mean total scores) were seen in the groups with either combined Eimeria/C. perfringens (group 1) or Paracox/C. perfringens (group 2) inoculations. The non-inoculated control animals generally had the lowest gut damage parameter scores. Differences in ileal sections were smaller, but the same trends as for duodenal and jejunal sections were detected. Figure 4 shows the mean total score of gut damage (sum of scores for villus fusion, congestion or dilation of blood vessels, capillary haemorrhages, red blood cells in the gut lumen, proteinaceous material in the gut lumen and epithelial cell defects) for jejunal sections in Trial 2. Accounting for the significant difference in the mean total scores between the observation days, a significant $(\mathrm{p}<0.01)$ difference between the groups was observed. This significant difference was situated between group 1 and groups 3,5 and 6.

\section{Discussion}

A model is presented in which a high percentage of the treated animals develop necrosis of the intestinal mucosa, but without induction of mortality and in which the gut is not overwhelmed by coccidial pathogens. The model uses an overdose of a live coccidiosis vaccine, and multiple oral $C$. perfringens inoculations. For the study of histopathological changes in necrotic enteritis induced by C. perfringens it is preferable not to have lesions caused by other agents. Inoculation of Paracox compared with the Eimeria field strain is thus preferable. Indeed, single E. maxima administration also induced gross gut lesions, in contrast to administration of a 10-fold dose of the coccidial vaccine. Furthermore, the $\mathrm{V} / \mathrm{C}$ ratio in birds receiving the single E. maxima inoculation was very 
low, illustrating that gut damage due to the inoculation was severe. The number of coccidial organisms replicating in the gut after E. maxima inoculation was very high a few days postinoculation, and this interferes with histological analysis. The above mentioned issues however do not undermine the relevance of using Eimeria inoculations for studying necrotic enteritis as seen in the field.

Quantification of the severity of necrotic enteritis in broilers is mostly done by lesion scoring or mortality rates (Riddell and Kong, 1992; Jackson et al., 2003; Williams et al., 2003). In experimental trials or field outbreaks not all animals develop lesions. In experimental trials also gross lesions are not always observed (Olkowski et al., 2006). Although in these cases, in some animals, microscopic analysis shows hyperaemia, haemorrhages and some epithelial cell defects, these lesions have not hitherto been quantified in a standardized protocol (Olkowski et al., 2006). In the present study, a scoring system was developed for lesions that are typically seen in the initial stages of necrotic enteritis, such as leakage of proteins into the gut lumen, and the presence of red blood cells in the lumen. The non-inoculated groups generally had lower total scores of gut damage parameters than those with single (Eimeria, Paracox, C. perfringens) inoculation, and generally single inoculations yielded lower total gut damage scores than those groups with combined $C$. perfringens/Paracox or C. perfringens/Eimeria maxima inoculations. This illustrates that the semiquantitative scoring system developed gives an indication of the severity of gut damage, even in the absence of grossly visible gut lesions. To use the scoring system in a reliable way, care should be taken over the selection procedure for sampling of gut sections. It is proposed that sampling of multiple specified regions (duodenum, jejunum and ileum), and not one single region, will yield the most reliable results. When comparing treatment groups without gross lesions it is clear that the location of gut sections to be sampled has to be identical between groups. In our study, the middle part of the second limb of the duodenum, the jejunal part proximal to the Meckel's diverticulum and the ileal section proximal to the ileo-caecal junction was chosen. The scoring system also showed that an overdose of live coccidial vaccine or $C$. perfringens itself induces gut damage, and that both 
act synergistically to induce necrosis. Thus the scoring system can be used to distinguish between treatment groups that do not develop macroscopic lesions, in experiments in which only a few experimental birds are used. Enumeration of intestinal C. perfringens organisms would also be a parameter that could be used to distinguish treatment groups that do not have gross lesions, but the reliability of enumeration in studying subclinical disease can be questioned since other bacteria and environmental factors can also possibly play a role. The histopathological scoring system can furthermore be used to evaluate the effect of other gut damage-inducing agents. Using the histopathological scoring system for experiments in which many birds develop gross lesions would not be an additional benefit, since macroscopic lesion scoring is sufficient in these cases.

Earlier gut damage caused by Eimeria spp., clearly predisposes to the development of $C$. perfringens-induced necrotic enteritis (Williams et al., 2003). E. maxima and a 10-fold dose of Paracox, combined with $C$. perfringens inoculations, both resulted in gut necrosis in the present trials, while single Eimeria, 10-fold dose Paracox or C. perfringens inoculations did not cause macroscopically visible necrotic lesions. The total score for gut damage parameters was however generally higher for single Eimeria, Paracox, or C. perfringens inoculated animals, compared with non-inoculated animals. Also villus length and the V/C ratio were lower in these groups compared with controls, illustrating that gut damage, although not grossly visible, was present in these groups. It can be hypothesized that gut damage caused by Eimeria or Paracox predisposes to the development of necrotic enteritis as it may allow easier migration of $C$. perfringens toxins in the gut wall when the epithelial barrier is affected. These toxins may have effects on many cell types, including endothelial cells, as seen in human gas gangrene (Bunting et al., 1997; Flores-Diaz et al., 2004). This may explain the hyperaemia and haemorrhages. The presence of cell debris and protein material in the lumen, to which $C$. perfringens is clearly attached, may enhance bacterial growth by the availability of essential amino acids. Indeed, $C$. perfringens is auxotrophic for more than 10 amino acids and is dependent on proteolysis and the supply of amino acids (Shimizu et al., 2002). Protein leakage to the gut lumen, caused by coccidia, could form a substrate for multiplication of 
the bacteria and subsequent induction of gut damage. Protein leakage could be one of the causes of growth retardation in broilers, together with decreases in villus length, and thus loss of absorptive surface. It is still not clear which Clostridium toxins induce the observed effects. While it was believed for more than 20 years that alpha toxin was the main cause of induction of gut lesions, recent studies provide strong arguments against this hypothesis. It was shown that isolates from clinically affected animals did not differ in the amount of alpha toxin produced in vitro when compared with isolates from healthy animals (Gholamiandehkordi et al., 2006), and an alpha toxin deletion mutant has been shown to induce similar severe necrotic lesions to those induced by its wild type parent strain (Keyburn et al., 2006).

The time point of Paracox administration does not seem to be a very important issue, provided that the inoculations are done at the same time or a few days before the $C$. perfringens inoculations. Indeed no differences in severity of lesions were observed when animals were inoculated with a 10-fold dose of Paracox 3 days before or in between $C$. perfringens inoculations.

In conclusion, a model is presented in which necrotic enteritis lesions caused by $C$. perfringens are reproduced without mortality of the animals, using an overdose of live coccidial vaccine strains. Furthermore, a method to analyse subtle microscopic alterations of the gut wall is proposed, in order to provide a semi-quantitative score of the gut damage caused by $C$. perfringens or other gut damage-inducing agents, even in conditions where no necrotic lesions are observed.

\section{Acknowledgements}

The excellent technical assistance of Marleen Foubert, Renzo Vercammen, Christian Puttevils and Delphine Ameye is greatly appreciated. Dr. R. Marshall (Veterinary Laboratories Agency, Weybridge, UK) is thanked for providing the Eimeria maxima strain. Gerard Huyghebaert (Institute for Agricultural and Fisheries Research, Melle, Belgium) is thanked for formulation of the feed. 
The Institute for Science and Technology (IWT), Flanders, is thanked for financial support. The authors thank the Ministry of Science, Research and Technology of Iran for financial support of the scholarship of the first author. Filip Van Immerseel is Postdoctoral Fellow of the Research Foundation - Flanders (FWO).

\section{References}

Bunting, M., Lorant, D.E., Bryant, A.E., Zimmerman, G.A., McIntyre, T.M., Stevens, D.L. \& Prescott, S.M. (1997). Alpha toxin from Clostridium perfringens induces proinflammatory changes in endothelial cells. Journal of Clinical Investigation, 100, 565-574.

Collier, C.T., van der Klis, J.D., Deplancke, B., Anderson, D.B. \& Gaskins, H.R. (2003). Effects of tylosin on bacterial mucolysis, Clostridium perfringens colonization, and intestinal barrier function in a chick model of necrotic enteritis. Antimicrobial Agents and Chemotherapy, 47, $3311-3317$.

Elwinger, K., Schneitz, C., Berndtson, E., Fossum, O., Teglof, B. \& Engstrom, B. (1992). Factors affecting the incidence of necrotic enteritis, caecal carriage of Clostridium perfringens and bird performance in broiler chicks. Acta Veterinaria Scandinavica, 33, 369-378.

Flores-Diaz, M., Thelestam, M., Clark, G.C., Titball, R.W. \& Alape-Girón, A. (2004). Effect of Clostridium perfringens phospholipase $\mathrm{C}$ in mammalian cells. Anaerobe, 10, 115-123.

Gholamaniandehkordi, A., Ducatelle, R., Heyndrickx, M., Haesebrouck, F. \& Van Immerseel, F. (2006). Molecular and phenotypical characterization of Clostridium perfringens isolates from poultry flocks with different disease status. Veterinary Microbiology, 113 , 143-152.

Jackson, M.E., Anderson, D.M., Hsiao, H.Y., Mathis, G.F. \& Fodge, D.W. (2003). Beneficial affect of beta-mannanase feed enzyme on performance of chicks challenged with Eimeria sp. and Clostridium perfringens. Avian Diseases , 47, 759-763. 
Kaldhusdal, M. \& Hofshagen, M. (1992). Barley inclusion and avoparcin supplementation in broiler diets. 2. Clinical, pathological, and bacteriological findings in a mild form of necrotic enteritis. Poultry Science, , 71, 1145-1153.

Kaldhusdal, M., Schneitz, C., Hofshagen, M. \& Skjerve, E. (2001). Reduced incidence of Clostridium perfringens-associated lesiosn and improved performance in broiler chickens treated with normal intestinal bacteria from adult fowl. Avian Diseases , 45, 149-156.

Keyburn, A.L., Sheedy, S.A., Ford, M.E., Williamson, M.M., Rood, J.I. \& Moore, R.J. (2006). Alpha-toxin of Clostridium perfringens is not an essential virulence factor in necrotic enteritis in chickens. Infection and Immunity, 74, 6496-6500.

Knarreborg, A., Simon, M.A., Engberg, R.M., Jensen, B.B. \& Tannock, G.W. (2002). Effects of dietary fat source and subtherapeutic levels of antibiotic on the bacterial community in the ileum of broiler chickens at various ages. Applied and Environmental Microbiology, 68, 5918-5924.

McReynolds, J.L., Byrd, J.A., Anderson, R.C., Moore, R.W., Edrington, T.S., Genovese, K.J., Poole, T.L., Kubena, L.F. \& Nisbet, D.J. (2004). Evaluation of immunosuppressant and dietary mechanisms in an experimental disease model for necrotic enteritis. Poultry Science, 83, 19481952.

Olkowski, A.A., Wojnarowicz, C., Chirino-Trejo, M. \& Drew, M.D. (2006). Responses of broiler chickens orally challenged with Clostridium perfringens isolated from field cases of necrotic enteritis. Research in Veterinary Science, 81, 99-108.

Riddell, C. \& Kong, X. (1992). The influence of diet on necrotic enteritis in broiler chickens. Avian Diseases , 36, 499-503.

Shimizu, T., Ohshima, S., Ohtani, K., Shimizu, T. \& Hayashi, H. (2001). Genomic map of Clostridium perfringens strain 13. Microbiology and Immunology, 45, 179-189.

Songer, J.G. (1996). Clostridial enteric diseases of domestic animals. Clinical Microbiology Reviews, 9, 216-234. 
Truscott, R.B. \& Al- Sheikhly, F. (1977).Reproduction and treatment of necrotic enteritis in broilers. American Journal of Veterinary Research, 38, 857-861.

Van Immerseel, F., De Buck, J., Pasmans, F., Huygebaert, G., Haesebrouck, F. \& Ducatelle, R. (2004). Clostridium perfringens in poultry : An emerging threat for animal and public health. Avian Pathology, 33(6), 537-549.

Wages, D.P. \& Opengart, K. (2003). Necrotic enteritis. In Y.M. Saif,, H.J Barnes, J.R. Glisson, A.M. Fadly, L.R. McDougald \& D.E. Swayne. (2003). Diseases of Poultry, 11th edn (pp. 781785). Ames: Iowa State Press.

Williams, R.B., Marshall, R.N., La Ragione, R.M. \& Catchpole, J. (2003). A new method for the experimental production of necrotic enteritis and its use for studies on the relationships between necrotic enteritis, coccidiosis and anticoccidial vaccination of chickens. Parasitology Research, 90, 19-26.

Williams, R.B. (2005). Intercurrent coccidiosis and necrotic enteritis of chickens: rational, integrated disease management by maintenance of gut integrity. Avian Pathology, 34(3), 159180. 
Table 1. Experimental design for Trials 1, 2 and 3 showing the days of age at which the challenges and vaccine were given and the days on which lesions were scored

\begin{tabular}{|c|c|c|c|c|c|}
\hline Trial & Group & $\begin{array}{l}\text { Clostridial } \\
\text { challenge } \\
\text { days }\end{array}$ & $\begin{array}{l}\text { Paracox-8 } \\
\text { vaccination } \\
\text { day }\end{array}$ & $\begin{array}{l}\text { E. maxima } \\
\text { challenge } \\
\text { day }\end{array}$ & $\begin{array}{c}\text { Lesion scoring } \\
\text { days }\end{array}$ \\
\hline 1 & 1 & $18,19,20,21$ & 19 & None & $18,21,25,27$ \\
\hline 1 & 2 & $18,19,20,21$ & None & None & $18,21,25,27$ \\
\hline 1 & 3 & None & 19 & None & $18,21,25,27$ \\
\hline 1 & 4 & None & None & None & $18,21,25,27$ \\
\hline 2 & 1 & $19,20,21,22$ & None & 20 & $19,22,23,24,25,26$ \\
\hline 2 & 2 & $19,20,21,22$ & 20 & None & $19,22,23,24,25,26$ \\
\hline 2 & 3 & $19,20,21,22$ & None & None & $19,22,23,24,25,26$ \\
\hline 2 & 4 & None & None & 20 & $19,22,23,24,25,26$ \\
\hline 2 & 5 & None & 20 & None & $19,22,23,24,25,26$ \\
\hline 2 & 6 & None & None & None & $19,22,23,24,25,26$ \\
\hline 3 & 1 & $19,20,21,22$ & 20 & None & $19,22,23,24,25,26$ \\
\hline 3 & 2 & $19,20,21,22$ & 16 & None & $\begin{array}{c}16,19,20,21,22,23,24,25 \\
26\end{array}$ \\
\hline 3 & 3 & $19,20,21,22$ & 20 & None & $19,22,23,24,25,26$ \\
\hline 3 & 4 & $19,20,21,22$ & 16 & None & $\begin{array}{c}16,19,20,21,22,23,24,25, \\
26\end{array}$ \\
\hline
\end{tabular}


Table 2. Broiler chicken finisher diets (percent w/w) for Trials 1, 2 and 3

\begin{tabular}{lcccc}
\hline Item & Trial 1 & Trial 2 & $\begin{array}{c}\text { Trial 3 } \\
\text { Group1 and 2 }\end{array}$ & $\begin{array}{c}\text { Trial 3 } \\
\text { Group 3 and 4 }\end{array}$ \\
\hline Wheat & & & & \\
Barley & 50.26 & 43.65 & 43.65 & 41.58 \\
Rye & 10 & 0 & 0 & 0 \\
Corn & 0 & 7.5 & 7.5 & 7.5 \\
Soybean meal & 5 & 0 & 0 & 0 \\
Soybeans & 0 & 0 & 0 & 18.89 \\
Herring meal & 0 & 5 & 5 & 5 \\
Wheat bran & 30 & 30 & 30 & 5 \\
Animal fat & 1.06 & 5 & 5 & 5 \\
Soy oil & 0 & 6.61 & 6.61 & 13.39 \\
Calcium carbonate & 1 & 1 & 1 & 1 \\
Dicalcium phosphate & 0.84 & 0 & 0 & 0.72 \\
Sodium chloride & 0 & 0 & 0 & 0.32 \\
Sodium bicarbonate & 0 & 0 & 0 & 0.24 \\
Lysine & 0.79 & 0.19 & 0.19 & 0 \\
Methionine & 0 & 0 & 0 & 0.08 \\
Threonine & 0 & 0 & 0 & 0.17 \\
Vitamin/mineral premix & 1 & 0 & 0 & 0.07 \\
& & 1 & 1 & 1 \\
\hline
\end{tabular}


Table 3. Macroscopic and microscopic lesion scoring system

\section{Macroscopic}

Focal necrosis and ulceration

Patches of necrosis 2 to $3 \mathrm{~cm}$ long

Diffuse necrosis typical of field cases $\quad 3$

\section{Microscopic lesions in the gut section}

\section{Villus fusion}

Occasional fusion of two villi in a section 1

Occasional fusion of more than two villi or several fusions of two 2

Multiple areas where more than two villi were fused 3

Large clusters of fused villi throughout 4

Dilation of capillaries

A few mildly dilated

Mildly dilated throughout

Moderately dilated throughout $\quad 3$

Severely dilated throughout

Capillary haemorrhage

A few red blood cells outside capillaries in some villi 1

A few red blood cells outside capillaries in most villi 2

Many red blood cells outside capillaries in parts of section 3

Severe haemorrhages throughout

Epithelial cell defects

Flattening of epithelial cells in a few villus tips $\quad 1$

Defect or micro-erosion at tips of a few villi 2

Defect or micro-erosion at tips of multiple villi 3

Severe erosions, large epithelial cell defects 4

Red blood cells gut lumen

A few

Some aggregates

Multiple aggregates

Whole lumen filled with aggregates

Proteinaceous material gut lumen

Some spots of material

Multiple spots of material

Very large clumps of material 
Table 4. Numbers of birds with macroscopic necrotic enteritis lesions in Trials 1, 2 and 3

\begin{tabular}{|c|c|c|c|c|c|c|c|c|c|c|}
\hline Trial & Group & Day & Day & Day & Day & Day & Day & Day & Total & Total \\
\hline & & 21 & 22 & 23 & 24 & 25 & 26 & 27 & & $\%$ \\
\hline \multirow[t]{4}{*}{1} & 1 & $0 / 3$ & $\mathrm{NS}^{\mathrm{a}}$ & NS & NS & $3 / 4$ & NS & $0 / 4$ & $3 / 11$ & 27.27 \\
\hline & 2 & $0 / 3$ & NS & NS & NS & $0 / 4$ & NS & $0 / 4$ & $0 / 11$ & 0 \\
\hline & 3 & $0 / 3$ & NS & NS & NS & $0 / 4$ & NS & $0 / 4$ & $0 / 11$ & 0 \\
\hline & 4 & $0 / 4$ & NS & NS & NS & $0 / 4$ & NS & $0 / 4$ & $0 / 12$ & 0 \\
\hline \multirow[t]{6}{*}{2} & 1 & NS & $0 / 6$ & $0 / 2$ & $0 / 4$ & $2 / 4$ & $0 / 4$ & NS & $2 / 20$ & 10 \\
\hline & 2 & NS & $0 / 5$ & $0 / 4$ & $1 / 3$ & $2 / 4$ & $2 / 5$ & NS & $5 / 21$ & 23.8 \\
\hline & 3 & NS & $0 / 5$ & $0 / 5$ & $0 / 3$ & $0 / 3$ & $0 / 3$ & NS & $0 / 19$ & 0 \\
\hline & 4 & NS & $0 / 3$ & $0 / 4$ & $0 / 4$ & $0 / 4$ & $0 / 7$ & NS & $0 / 22$ & 0 \\
\hline & 5 & NS & $0 / 4$ & $0 / 4$ & $0 / 4$ & $0 / 4$ & $0 / 7$ & NS & $0 / 23$ & 0 \\
\hline & 6 & NS & $0 / 4$ & $0 / 3$ & $0 / 4$ & $0 / 4$ & $0 / 5$ & NS & $0 / 20$ & 0 \\
\hline \multirow[t]{4}{*}{3} & 1 & NS & $0 / 3$ & $2 / 4$ & $5 / 6$ & $4 / 6$ & $5 / 7$ & NS & $16 / 26$ & 61.53 \\
\hline & 2 & $0 / 3$ & $1 / 3$ & $2 / 4$ & $1 / 3$ & $1 / 3$ & $1 / 4$ & NS & $6 / 20$ & 30 \\
\hline & 3 & NS & $0 / 3$ & $0 / 4$ & $2 / 5$ & $3 / 6$ & $3 / 6$ & NS & $8 / 24$ & 33.33 \\
\hline & 4 & $1 / 3$ & $3 / 3$ & $0 / 4$ & $1 / 3$ & $3 / 4$ & $2 / 4$ & NS & $10 / 21$ & 47.61 \\
\hline
\end{tabular}

${ }^{a}$ Not scored

On day 18 in trial 1, day 19 and trial 2, and day 16 and 19 in trial 3 no necrotic enteritis lesions were detected. 
Table 5. Mean macroscopic necrotic enteritis lesion scores in Trial 3

\begin{tabular}{|c|c|c|c|c|c|c|c|c|c|c|c|c|c|c|c|c|c|c|c|c|c|}
\hline \multirow[b]{2}{*}{ Group } & \multicolumn{3}{|c|}{ Day 21} & \multicolumn{2}{|c|}{ Day 22} & \multicolumn{4}{|c|}{ Day 23} & \multicolumn{3}{|c|}{ Day 24} & \multicolumn{3}{|c|}{ Day 25} & \multicolumn{3}{|c|}{ Day 26} & \multicolumn{3}{|c|}{$\begin{array}{l}\text { Total means }{ }^{\mathrm{d}} \\
\text { (days 22-26) }\end{array}$} \\
\hline & $\mathrm{D}^{\mathrm{a}}$ & $\mathrm{J}^{\mathrm{b}}$ & $\mathrm{I}^{\mathrm{c}}$ & $\mathrm{D}$ & $\mathrm{J}$ & I & D & $\mathbf{J}$ & I & $\mathrm{D}$ & $\mathrm{J}$ & I & $\mathrm{D}$ & $\mathrm{J}$ & I & $\mathrm{D}$ & $\mathrm{J}$ & I & $\mathrm{D}$ & $\mathbf{J}$ & I \\
\hline 1 & $\mathrm{NS}^{\mathrm{e}}$ & NS & NS & 0 & 0 & 0 & 0 & 0.5 & 0 & 1.17 & 2.17 & 1.11 & 0.17 & 0.67 & 0.67 & 0.29 & 1.14 & 0.43 & 1.63 & 4.48 & 2.21 \\
\hline 2 & 0 & 0 & 0 & 0 & 0.33 & 0 & 0.5 & 0 & 0 & 0 & 0.33 & 0 & 0 & 0.33 & 0 & 0.25 & 0.5 & 0.25 & 0.75 & 1.49 & 0.25 \\
\hline 3 & NS & NS & NS & 0 & 0 & 0 & 0 & 0 & 0 & 0.8 & 1 & 0 & 0.5 & 0.5 & 0.33 & 0.83 & 1 & 0.33 & 2.13 & 2.5 & 0.66 \\
\hline 4 & 0 & 0.33 & 0 & 0.66 & 1.33 & 0 & 0 & 0 & 0 & 0 & 0.33 & 0 & 0 & 0.75 & 0.25 & 0.5 & 0.75 & 0.25 & 1.16 & 3.49 & 0.5 \\
\hline
\end{tabular}

${ }^{\mathrm{a}}$ duodenum; ${ }^{\mathrm{b}}$ jejunum; ${ }^{\mathrm{c}}$ ileum; ${ }^{\mathrm{d}}$ day 21 not included since no scores for group 1 and $3 ;{ }^{\mathrm{e}}$ not scored.

All groups given a 10-fold dose of Paracox-8, at day 16 for groups 2 and 4 and day 20 for groups 1 and 3; challenged with $C$. perfringens strain 56 o three times daily n days 19,20, 21 and 22; groups 1 and 2 fed a wheat-rye based diet with high fishmeal content (30\%), groups 3 and 4 fed a low fishmeal content ( $5 \%$ ) diet. 
Table 6. Villus length/crypt depth ratio ${ }^{a}$ (V/C ratio) for duodenal and jejunal sections in Trial 1

\begin{tabular}{|c|c|c|c|c|}
\hline \multirow[b]{2}{*}{ Day } & \multicolumn{4}{|c|}{ Group } \\
\hline & 1 & 2 & 3 & 4 \\
\hline 21 & $8.87^{\mathrm{a}}$ & $9.77^{\mathrm{AB}}$ & $11.19^{\mathrm{AB}}$ & $13.28^{\mathrm{B}}$ \\
\hline 25 & $2.40^{\mathrm{A}}$ & $10.34^{\mathrm{B}}$ & $6.80^{\mathrm{C}}$ & $10.66^{\mathrm{B}}$ \\
\hline \multirow[t]{2}{*}{27} & $5.75^{\mathrm{A}}$ & $6.67^{\mathrm{A}}$ & $7.77^{\mathrm{A}}$ & $12.14^{\mathrm{B}}$ \\
\hline & \multicolumn{4}{|c|}{ Group } \\
\hline Day & 1 & 2 & 3 & 4 \\
\hline 21 & $3.62^{\mathrm{A}}$ & $4.05^{\mathrm{A}}$ & $4.66^{\mathrm{A}}$ & $6.68^{\mathrm{B}}$ \\
\hline 25 & $2.70^{\mathrm{A}}$ & $5.22^{\mathrm{B}}$ & $5.78^{\mathrm{B}}$ & $5.65^{\mathrm{B}}$ \\
\hline 27 & $3.90^{\mathrm{A}}$ & $2.43^{\mathrm{A}}$ & $4.83^{\mathrm{A}}$ & $8.81^{\mathrm{B}}$ \\
\hline
\end{tabular}

${ }^{a}$ random measurement of 10 villi and crypts per gut section by a PC-based image analysis system. Values with different upper case superscripts are statistically significant different $(P \leq 0.05)$ 
Table 7. Villus length/crypt depth ratio ${ }^{a}$ (V/C ratio) for duodenal, jejunal and ileal sections in Trial 2

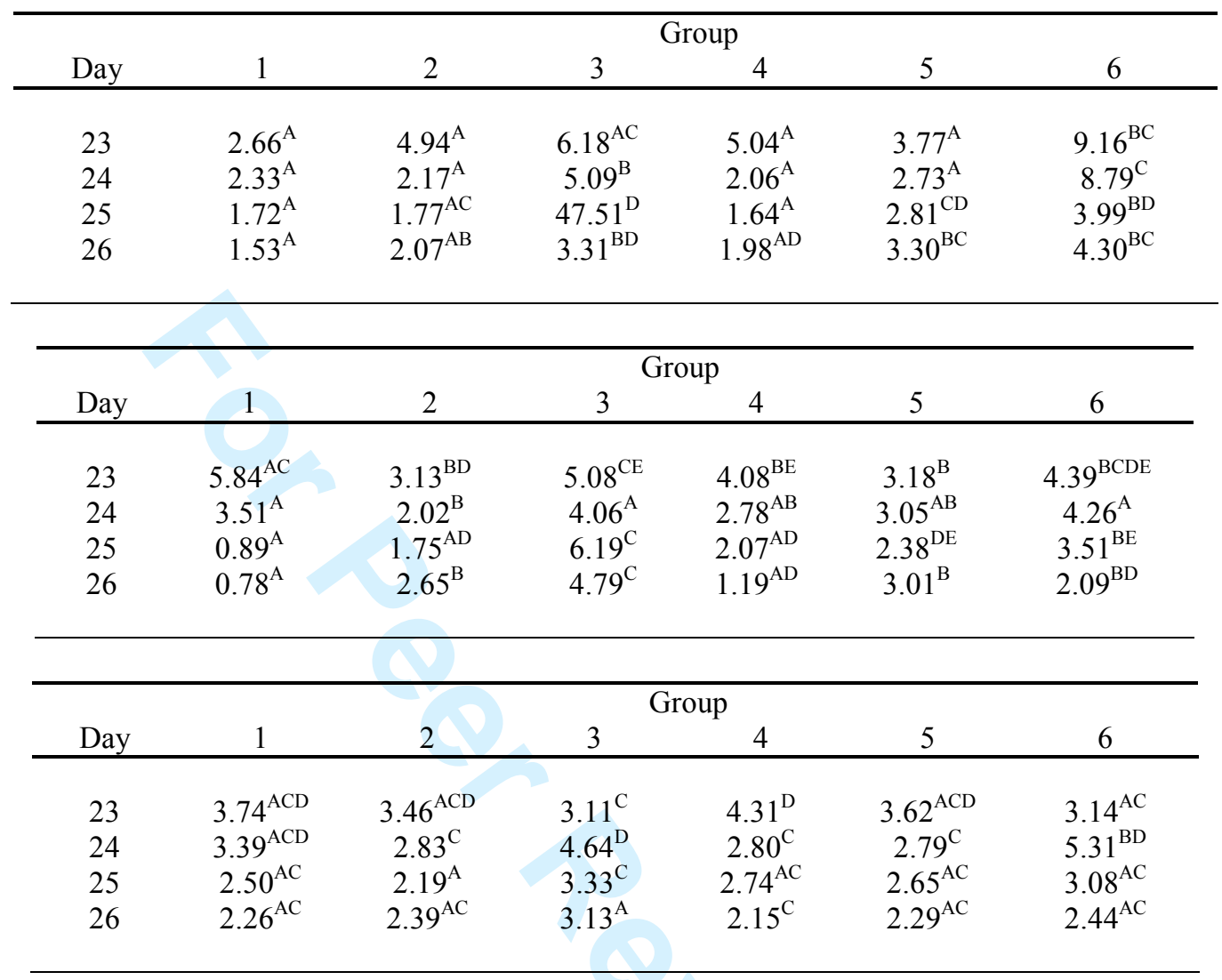

${ }^{a}$ random measurement of 10 villi and crypts per gut section by a PC-based image analysis system.

Values with different upper case superscripts are statistically significant different $(P \leq 0.05)$ 


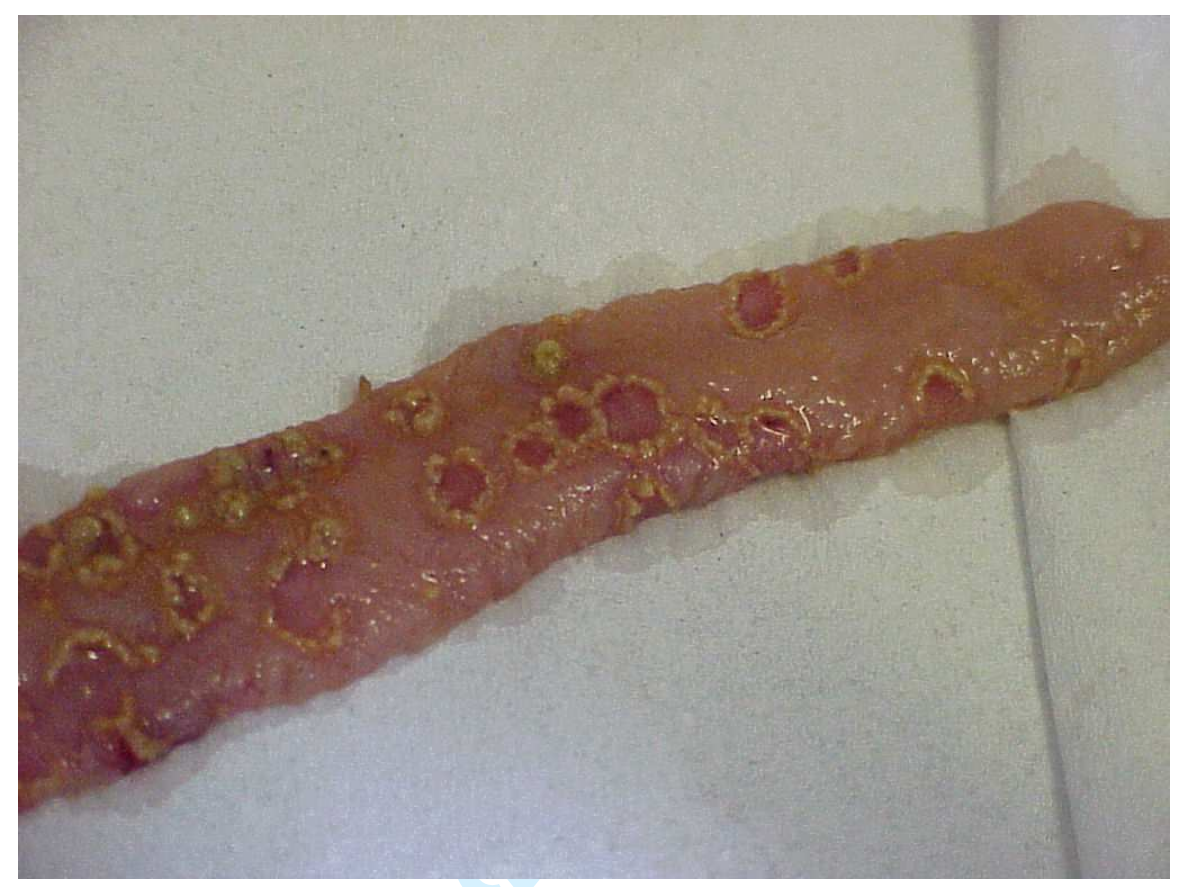

Figure 1. Focal necrosis of the jejunum of broiler chickens at day 25 , after oral challenge three times a day (9 a.m., 1 p.m. and 5 p.m.) with about $4 \times 10^{8}$ cfu $C$. perfringens strain 56 bacteria at day 18,19,20 and 21, and oral challenge with a ten-fold dose of Paracox at day 19.(Trial 1, Group 1).

Deleted: jejunum of broiler chickens that were orally challenged three times a day ( 9 a.m., 1 p.m. and 5 p.m.) with about $4 \times 10^{8}$ cfu $C$. perfringens strain 56 bacteria at day $18,19,20$ and 21 , and with a ten-fold dose of Paracox at day 19. 


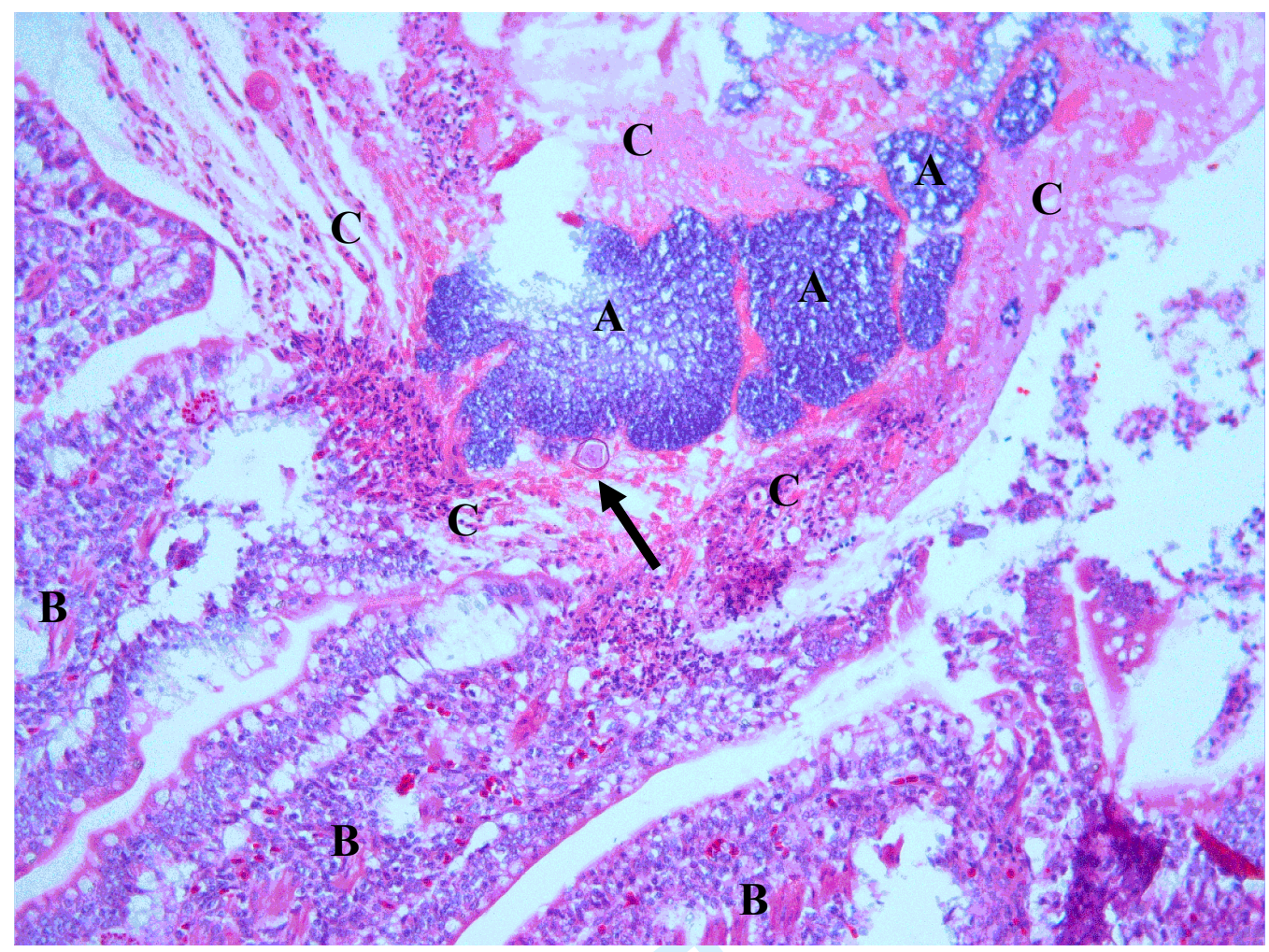

Figure 2. Necrotic enteritis lesions, typically visible as severe necrosis of the intestinal mucosa, with an abundance of fibrin admixed with cellular debris adherent to the necrotic mucosa, in which large clusters of bacteria are present. Also marked infiltration of heterophilic granulocyes in the gut wall is observed. The arrow points to an oocysts of Eimeria spp. Both pictures are derived from jejunal sections after combined Paracox/C. perfringens inoculations in trial 3 (A : C. perfringens bacteria; B : villi; C: cellular debris/fibrin) 


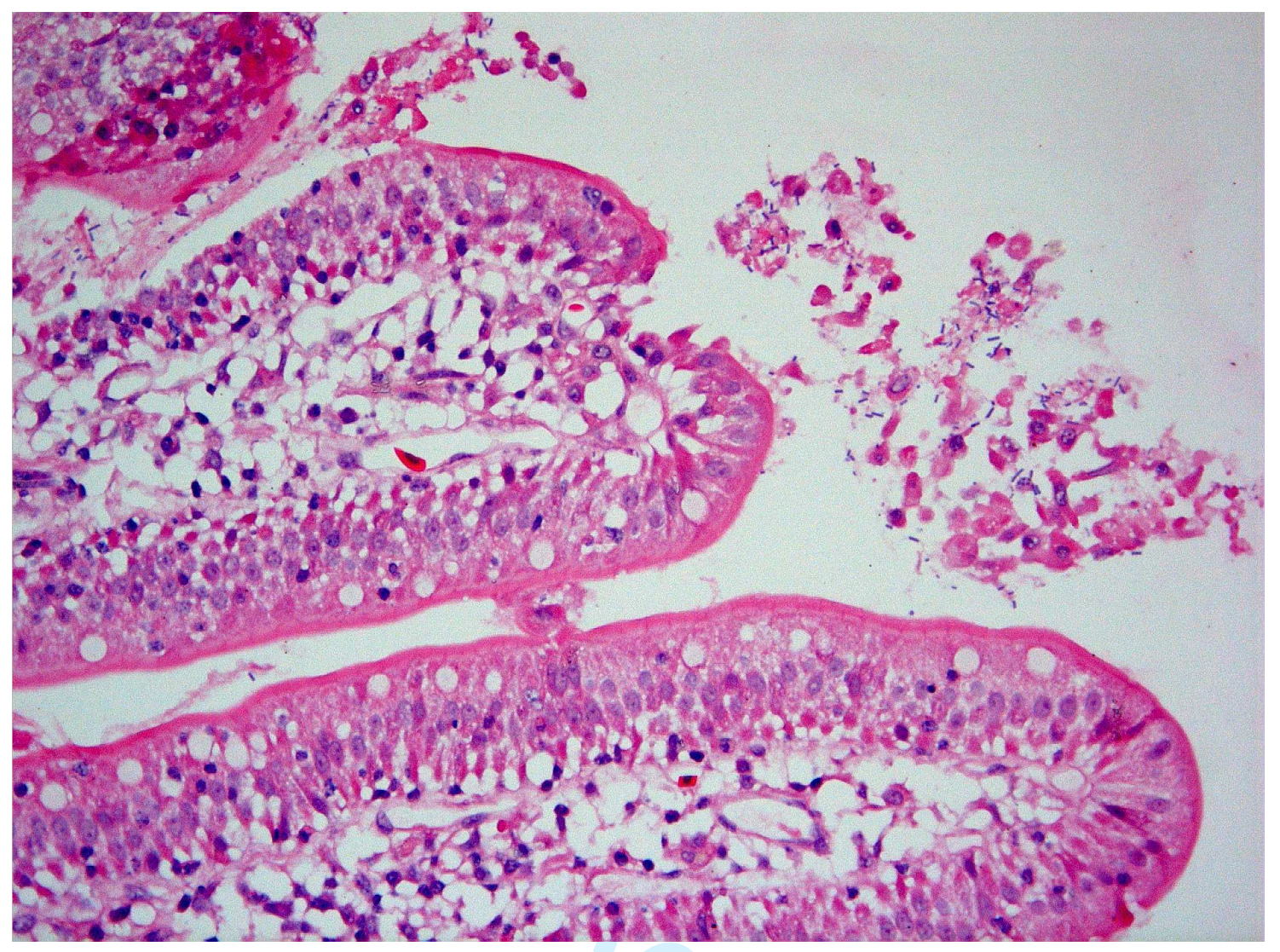

Figure 3. In zones without necrotic lesions, Clostridium perfringens bacteria attach to cell debris and fibrin-like material in the lumen. 


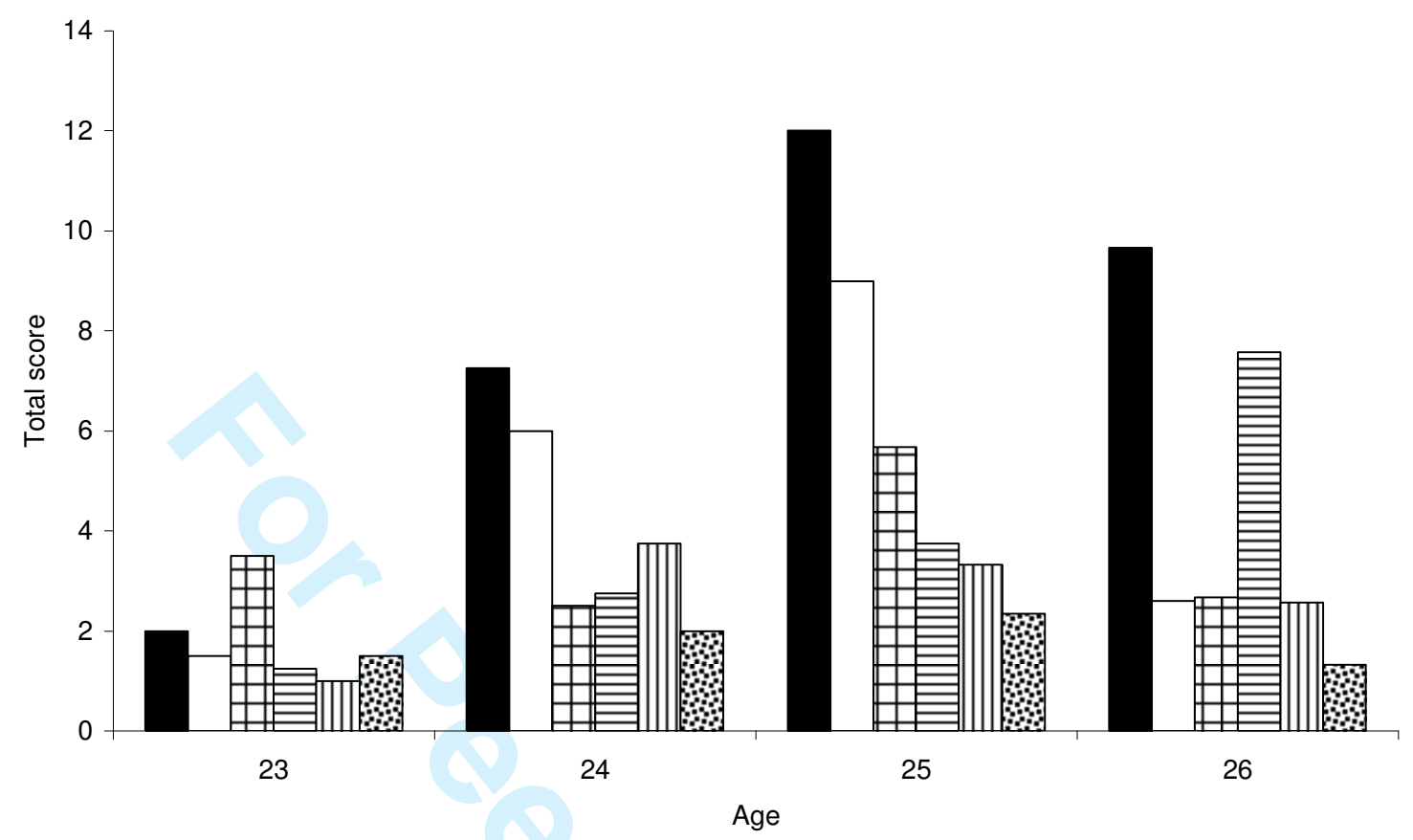

Figure 4. Total score of gut damage during time. Results are shown for jejunal sections in trial 2. Animals were non-inoculated (bars containing dots), inoculated with a ten-fold dose of Paracox at day 20 (bars containing vertical lines), inoculated with E. maxima at day 20 (bars containing horizontal lines), inoculated with Clostridium perfringens at days 18, 19, 20 and 21 (bars containing squares) or inoculated with both E. maxima/C. perfringens (black bars) or both Paracox/C. perfringens (transparent bars). The total score of gut damage was calculated as the sum of the mean scores for villus fusion, congestion, capillary haemorrhages, red blood cells in lumen, epithelial defects and protein material in lumen, as described in the materials and methods section. One jejunal section was analyzed for each sampled animal. The number of sampled animals can be consulted in Table 2. 